\title{
Multi-resolution eddy detection from ocean color and sea surface temperature images
}

\author{
Imen Karoui $^{1,2}$, Hervé Chauris ${ }^{2}$, Pierre Garreau ${ }^{1}$, and Phillipe Craneguy ${ }^{3}$ \\ ${ }^{1}$ Ifremer, dyneco, Technopôle Brest Iroise, 29280 PLOUZANE, France \\ Email: imen.karoui@ifremer.fr,pierre.garreau@ifremer.fr \\ ${ }^{2}$ Centre de Géosciences, MINES-ParisTech \\ Email: herve.chauris@mines-paristech.fr \\ ${ }^{3}$ Actimar, 36 quai de la douane 29200 Brest, France \\ Email: craneguy@actimar.fr
}

\begin{abstract}
We propose a multi resolution method for automatic detection of eddies on both simulated and satellite Sea Surface Temperature (SST) or ocean color images. Our approach consists in a curvelet based analysis of image fronts along circles with specified radius values. The main interests of the proposed method are:

- It is carried out on image gradient, and is thus not sensitive to the choice of binarization threshold;

- With the multi-resolution aspect and the anisotropic spatial support shape of curvelet elements, we can deal with no perfectly circular eddies (curvelet width) and we also take into account the image regularity to distinguish between real eddies and noise (curvelet length).
\end{abstract}

We have tested the method on several modeled and satellite images in the North Western Mediterranean sea and the results prove the relevance of the proposed approach.

\section{INTRODUCTION}

In coastal oceanography, sub-mesoscale structures, e.g. eddies in the range of 20 to $100 \mathrm{~km}$, appear to be a key element to better understand the surface circulation and velocities, but also the marine ecosystem as eddies may drive phytoplankton blooms or larvae. For simulated data, the associated velocity field or sea surface elevation are certainly the most relevant dynamical variable for eddy detection. But for remote sensing data, such small eddies are not caught by altimetry or in situ measurements. However, tracers (e.g. Sea Surface Temperature images (SST) or ocean color) in a turbulent ocean may reveal the sub-mesoscale circulation.

Such mesoscale eddy detection allows for a statistical evaluation of the numerical modeling performance in terms of eddy positions, sizes and motion and so is a key step for subsequent data assimilation.

Several methods extracting surface signature on SST or ocean color images are proposed on the literature [7]-[10], [15]. Most of these approaches describe eddies as circular structures on image gradient. In this paper, we also describe eddies as circular SST/ocean color fronts but we propose some solutions to some drawbacks raised by classical approaches mainly the choice of the binarization thresholds for edge extraction and the high rate of false detections due to noise and to the non circular shape of some SST/ocean color fronts. The proposed method introduces some additional features about eddy boundaries smoothness and allows some flexibility about eddy shapes.

The paper is organized as follows. A brief state of the art about eddy detection from SST/ocean color images is given in section II. The principle of curvelet transform is described in section III. The eddy detection algorithm is detailed in section IV. Experiments are reported and discussed in section V and conclusions are drawn in section VI.

\section{EdDy DETECTION: STATE OF THE ART}

Eddies correspond to water masses with a rotating component around the same pivoting center [1]. In the case of simulated data, the associated velocity field or sea-surface elevation is certainly the most relevant dynamical variable for describing eddies. The most popular features are (1) the Okubo-Weiss criterion [2] that aims at separating the velocity field into regions of high vorticity and strain, and (2) the Lyapunov exponents that quantify local stirring [5].

For remote sensing images, the velocity field is however not available and other techniques have been developed to automatically detect mesoscale eddy structures. They assume a high correlation between the SST/ocean color and the velocity field [6] and eddies are mainly described on SST/Ocean color image gradient as more or less circular structures.

Eddy detection algorithms are mainly carried out on spatial or temporal image gradients and can be divided into three main approaches:

1) Texture-based approaches: These methods are based on the extraction of eddy signatures from gradient orientation map [11]-[14]. In [14], the authors compute the singularity map by processing the wavelet projections on the modulus of the gradients. Singularity analysis on SST is then used to uncover the circulation patterns in global ocean. In [11], [13], eddy detection is based on curvature estimation;

2) Shape fitting based methods: These geometrical approaches mainly consist in matching a given shape (circles, ellipses, circle arcs, ...) with image edges. The Hough transform, least mean square error technique and several other circle or ellipse fitting algorithms have been 


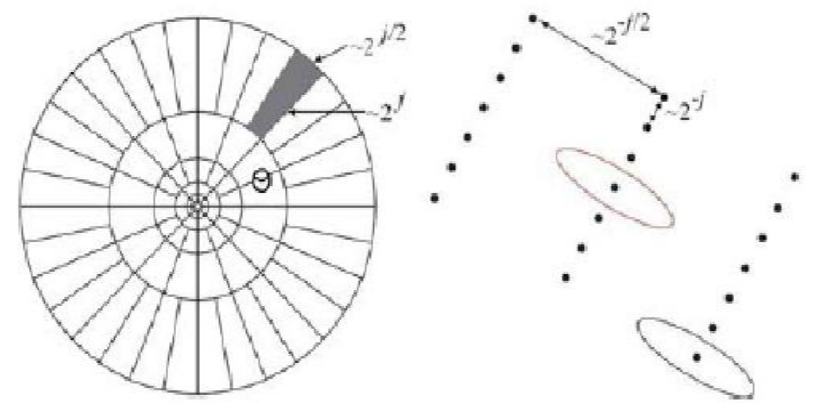

Fig. 1. Curvelets in frequency domain (left) and spatial domain (Left) [4].

applied to determine the radius and the central position of eddies on binary edge maps [7], [8];

3) Automatic machine learning based approaches. These methods are based on a learning scheme, mainly Artificial Neural Network (ANN) classifiers that analyze the neighboring pixels to distinguish between eddies and non eddy structures [9], [10], [15].

Each eddy detection category has some drawbacks. Oriented texture based methods give a high rate of false detection [13]. Geometrical methods for detecting circles or more complicated shapes are usually applied on binary images obtained after segmentation. They are thus very sensitive to the selected threshold values. And automatic supervised techniques needs a reliable manually segmented data base. They are restricted to eddy center detection and are always followed by a geometrical method to estimate the eddy radius.

\section{Curvelet transform}

Curvelet, proposed by E. Candès and D. Donoho [4], is an effective framework to represent edges and other singularities along curves much more efficiently than the traditional wavelet based transforms. This transform is designed to represent images at different scales and different angles. In figure 1, we show curvelets on both frequency and spatial domain. What is attractive with curvelet transform for our application is the elliptical shape of curvelet support in spatial domain with the parabolic scaling law between the width $\left(2^{-j}\right)$ and length $\left(2^{-j / 2}\right)$ of the curvelet basis elements. This parabolic scaling law induces an anisotropic elongated needle shaped structures. This shape is suitable to describe curves which are smooth away from discontinuities. Such functions fit natural images especially SST/ocean images where discontinuities are generated by edges (SST/ocean color fronts) and where edges are often gathered along smooth boundaries of image objects (for our case eddy contours). FigureS 3 and 4 show the effectiveness of curvelet to represent eddy contours and to describe edge regularity according to a specified orientation. Multi resolution transform allow us to analyze eddy boundaries with different curvelet basis element sizes and orientations. So using different scales we can characterize eddy boundaries continuity and smoothness (figure 4) .

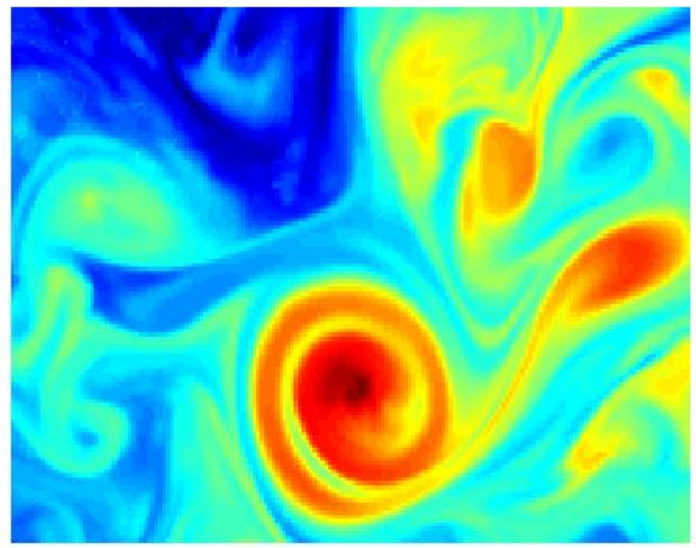

Fig. 2. Simulated SST image

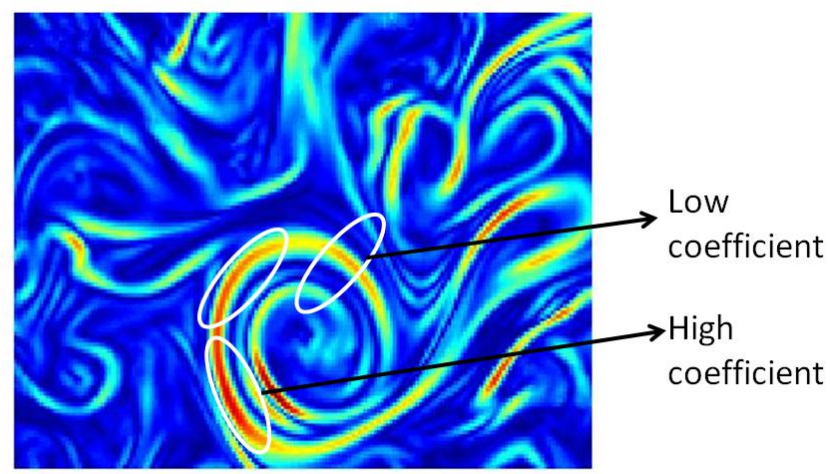

Fig. 3. Curvelet coefficients for different orientations of some SST fronts of image of Fig. 2

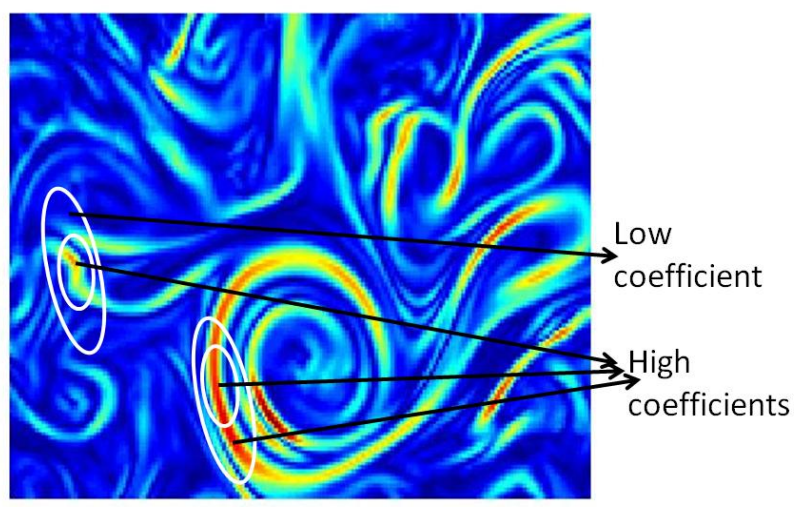

Fig. 4. Curvelet coefficients for different scales of some SST fronts of image of Fig. 2.

Here, we use the wrapping implementation of the digital curvelet transform [3]. 


\section{METHOD DESCRIPTION}

We aim at detecting on SST/ocean color remote sensing and simulated images, eddies with specified radius range. Our approach is based on the interesting properties of curvelet described in Section II. In fact, we consider eddies as nearly circular structures with high contract across eddy contours and with high smoothness along eddy boundaries. The proposed method consists in computing a punctual likelihood for each image pixel. This likelihood evaluates the pixel probability to be a center of an eddy with a specified radius $R$. We note this likelihood by $\Pi_{R}(i, j)$, it is expressed as follows:

$$
\Pi_{R}(i, j)=\sum_{s=1}^{S} \oint_{C_{(i, j), R}} \operatorname{curv}_{s, \theta(x, y)}^{2}(x, y)
$$

where $s$ is the scale index, $C_{(i, j), R}$ is the circle of radius $R$ and center $(i, j), \theta(x, y)$ is the direction of the tangent to $C_{(i, j), R}$ at the pixel $(x, y)$ and $\operatorname{curv}_{s, \theta(x, y)}$ the curvelet coefficient computed for scale $s$ and orientation $\theta(x, y)$.

Figure 5 shows some curvelets computed for different scales along a circle with a given radius.

Our algorithm consists of the following main steps:

1) Image gradient computation and normalization

2) Likelihood computation for the specified radii according to Eq.1

3) Selection of the highest coefficients

\section{RESULTS AND DISCUSSION}

We have tested the method on several SST/ocean color results of hydrodynamical eddy resolving modeling and satellite images in the North Western Mediterranean sea. For these zones we are interested in detecting only oceanic eddy structures of size range from 20 to $60 \mathrm{~km}$.

Model result images are not noisy and have a great number of eddies with different radii. Some eddies are easily detectable and are quite circular with well contrasted boundaries (for example eddy centers marked by $E$ in the figure 6). Others are more difficult to distinguish because they have deformed shapes and blurry edges. They often correspond to eddies at the beginning or the end of life (for example eddy centers marked by $D$ in the figure 6).

In figure 7, we show the result of the application of the proposed method using 4 scales and radii of 30,40 and $50 \mathrm{~km}$. Hydrodynamical eddy resolving models generate in addition to SST/ocean color images, Sea Surface Height (SSH) maps. As dynamically cyclonic and anti-cyclonic eddies appear as positive respectively negative sea level anomaly, these SSH maps can serve as a ground truth map to evaluate the performances of the proposed method.

According to figure 7 we can see that the most detected circles correspond to reliable eddies even they are not very visible on SST map. Some detected circles are more or less spatially shifted with respect to real eddy position. This lack of accuracy can be explained by the not perfect circular shape of eddies, the fact that the SST surface signature is not necessary

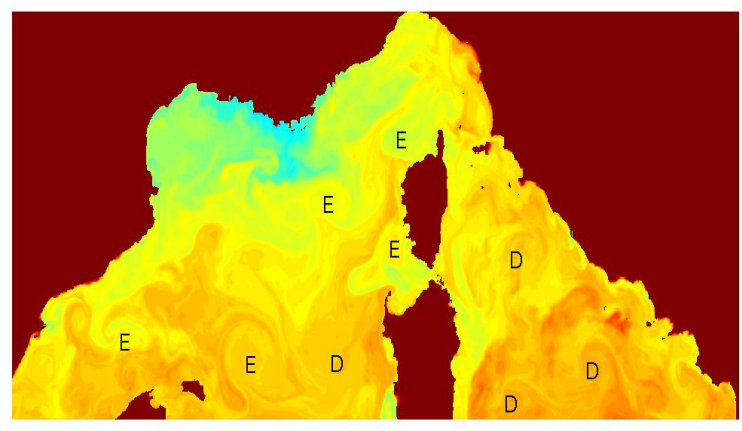

Fig. 6. Model result SST image (september 2007): eddy centers marked by $E$ correspond to well contrasted eddies and eddy centers marked by $D$ correspond to deformed or not contrasted eddies.

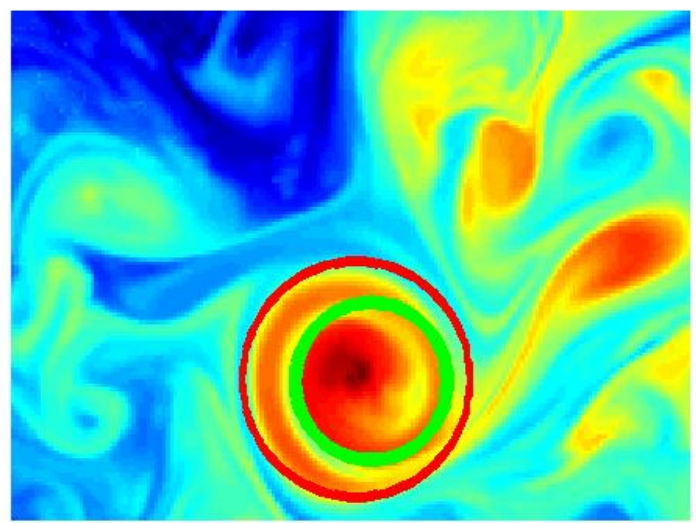

Fig. 9. Detected circles on SST map $R \in\{20,30 \mathrm{~km}\}$.

centered at the driving eddy and also by the multi resolution eddy front analysis.

In figures 8 and 9, we show some results carried out on two hydrodynamical eddy resolving modeling result maps. In figure 8, we present the detected circles on a SST model result image of North Western Mediterranean sea taken two days after the image of figure 7 .

In figure 9, we show a detected cyclonic spiral eddy on a simulated SST image (Balears front). The detection result provides a family of circles that fit the spiral shape of the filament.

In figures 10,11 and 12, we show results obtained for a set of remote sensing maps (respectively Catalan shelf, Alboran sea and Algerian currents) taken in summer 2006. These images contain noisy and blurred edge eddies compared to images coming from models and are mainly covered by clouds.

For both results of models and remote sensing images, almost well contrasted and also less contrasted eddies are detected by the proposed method. The method fails at detecting eddies with very deformed shape for example in figure 12 the eddy (presented by a dashed line (figure 12 (a)) has very 

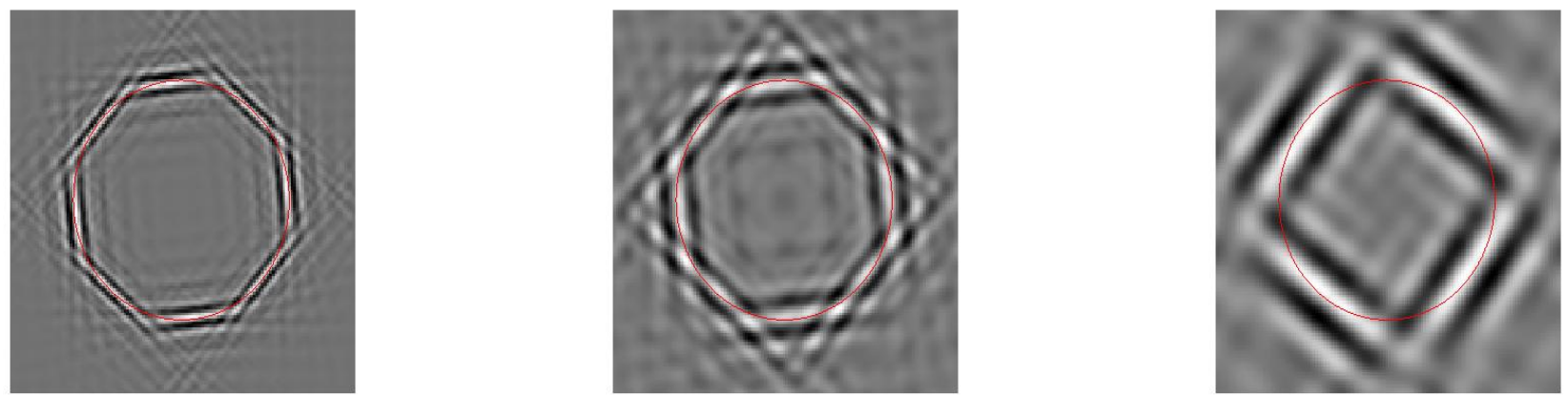

Fig. 5. Curvelets computed from different scales along a circle with a given radius : from finer (left) to coarser scale (right)).

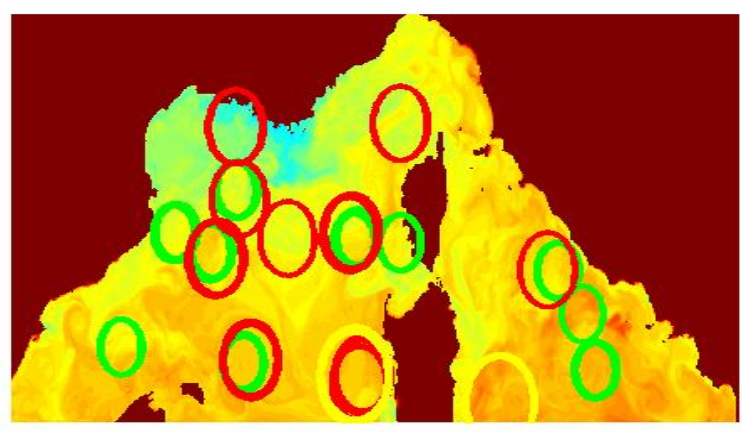

(a) Detected circles plotted on SST map

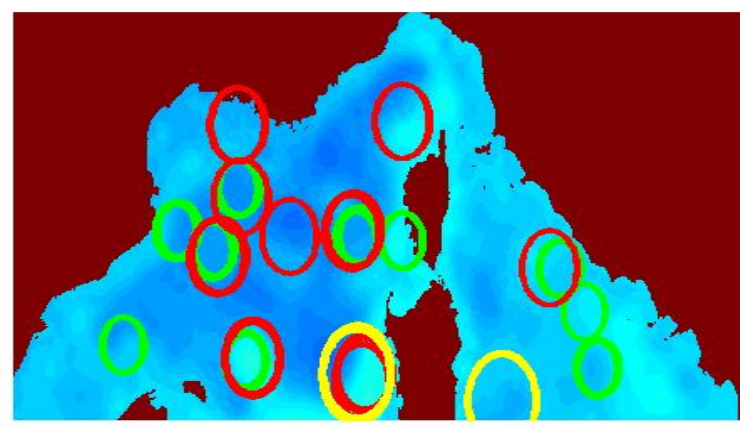

(b) Detected circles plotted on SSH map

Fig. 7. Detected circles of Fig.6 $R \in\{30,40,50 \mathrm{~km}\}$.

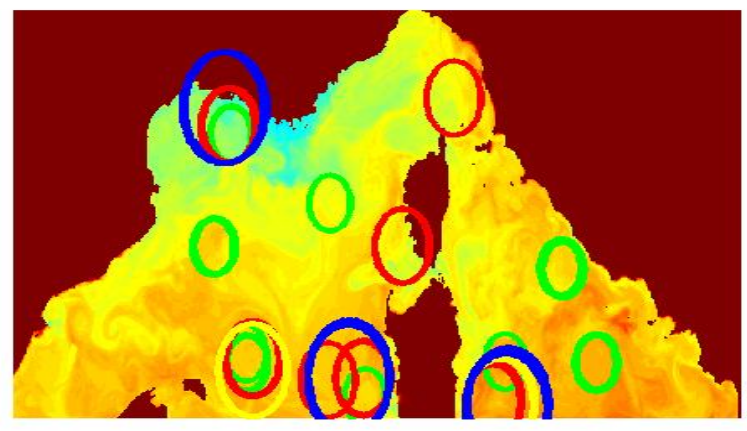

(a) Detected circles plotted on SST map

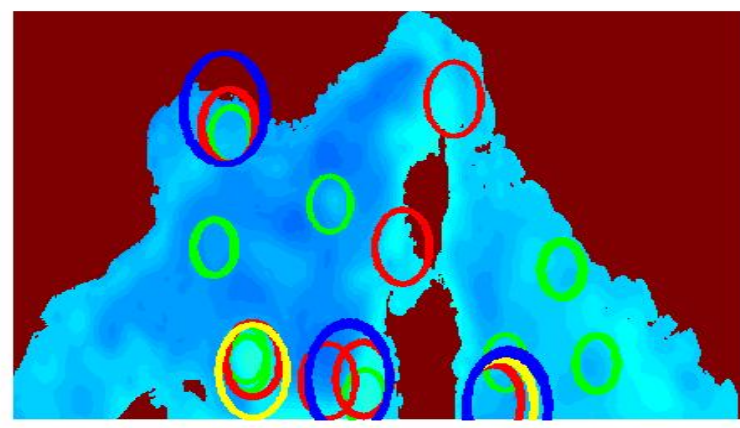

(b) Detected circles plotted on SSH map

Fig. 8. Detected circles, $R \in\{20,30,40,50 \mathrm{~km}\}$.

deformed shape. During his life this eddy will be detected for example 2 days after in figure 12 (b) where eddies have not circular shape but its boundaries are more contrasted: the method detects a set of circles that fit the eddy boundaries.

The number of the detected eddies depend on the chosen threshold. The choice of this threshold can be fixed by the user if we have an idea of the number of the expected reliable eddies. Eddies have a quite long life period (some days to some week for large eddies). So, applying the detection method on a large image sequence may help the elimination of false detection cases generated by a low threshold.

\section{CONCLUSION}

We proposed a robust method for the detection of oceanic surface eddies. The method analysis SST/ocean color fronts in a multi resolution scheme using curvelet transform. The curvelet, well suited to fit natural curves thanks to its spatial support and its several orientations, allows us to take into account in addition to the edge strength, eddy boundaries 


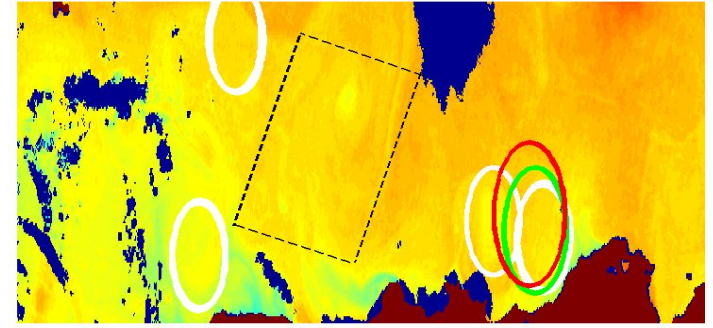

(a) Detected circles on remote sensing SST image $R \in\{20,30,40 \mathrm{~km}\}$

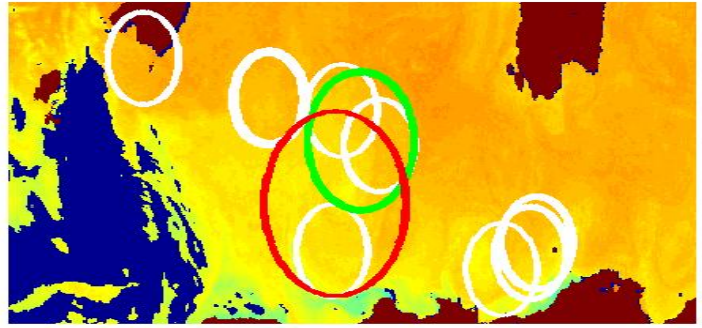

(b) Detected circles on remote sensing SST image $R \in\{40,60,80 \mathrm{~km}\}$.

Fig. 12. Detected eddies by the proposed method (Algerian current).

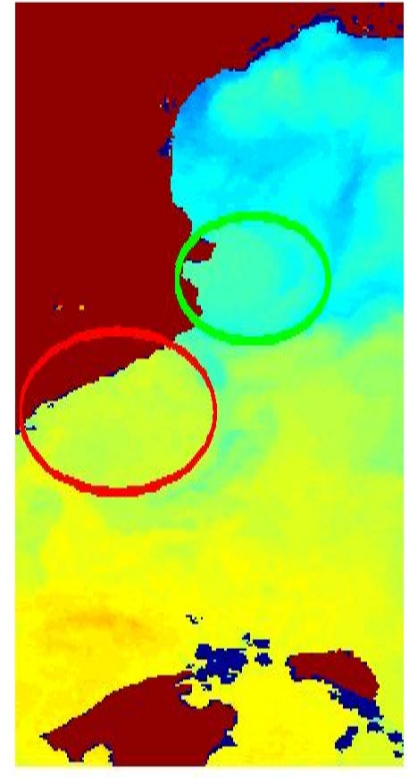

Fig. 10. Detected circles on remote sensing SST image (NOAA, Catalan shelf) $R \in\{35,45 \mathrm{~km}\}$.

smoothness and convexity. The results show the relevance and the effectiveness of the proposed approach.

This eddy detection algorithm allows us deeper analysis of detected eddies not only according to their size but also according to their motion and their polarization (cyclonic or anticyclonic). The application of this method to larger set of data for larger time periods are now in progress. And a tracking method based on this detection scheme is currently under development.

\section{REFERENCES}

[1] C. Pasquero, A. Provenzale and A. Babiano, "Parameterization of dispersion in two-dimensional turbulence", J. Fluid Mech. Volume 439, Page(s): 279-303, 2001.

[2] J.B. Weiss, "The dynamics of entrophy transfer in two-dimensional hydrodynamics”.IPhysica D. Volume 48. Page(s): 273—294, 1991.

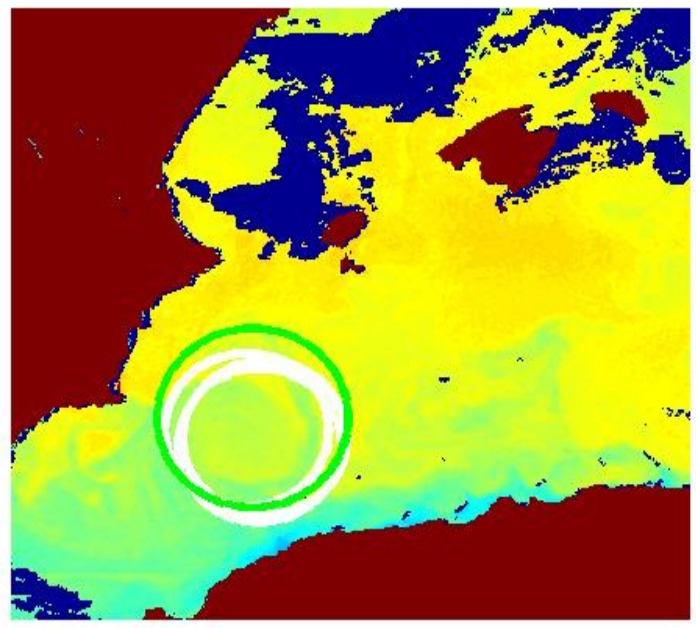

Fig. 11. Detected circles on remote sensing SST image (NOAA, Alboran sea) $R \in\{35,45 \mathrm{~km}\}$.

[3] E.J. Candes, L. Demanet D. L. Donoho, and L. Ying, "Fast discrete curvelet transforms".Applied and Computiational Mathematics, Caltech, Tech. Rep., 2005.

[4] E.J. Candes, and L. Donoho, "Curvelets: A surprisingly effective nonadaptive representation of objects with edges". Curves and Surface, Vanderbilt University Press, Nashville, TN. Page(s): 123-294143, 1999.

[5] T. Koh and B. Legras, "Hyperbolic lines and the stratospheric polar vortex". Chaos, Volume 12. Page(s): 382-394, 2002.

[6] T. Sugimura, S. Tanaka, and Y. Hatakeyama "Surface temperature and current vectors in the sea of Japan from NOAA-7 AAVHRR data". Remote sensing shelf sea hydrodyn. Proc. 15 Int. Liege. Colloq. Page(s): 133147, 1984.

[7] S.H. Peckinpaugh and R.J. Holyer, "Circle detection for extracting eddy size and position from satellite imagery of the ocean". IEEE Transactions on Geoscience and remote sensing. Volume 32. Page(s): 267-273, 1994.

[8] A. Fernandes and S. Nascimento, "Automatic Water Eddy Detection in SST Maps Using Random Ellipse Fitting and Vectorial Fields for Image Segmentation”. Lecture Notes in Computer Science. Volume 4265. Page(s): 77-88, 2006.

[9] M. Castellani, "Automatic detection of Mediterranean water eddies from satellite imagery of the Atlantic Ocean". Proc. of the 1st Virtual International Conference on Intelligent Production Machines and Systems, Pham, D. T., Eldukhri, E. E. and Soroka, A. J. (eds), Elsevier (Oxford) Page(s): 168-170, 2005.

[10] J. Hai, Y. Xiaomei, G. Jianming and G. Zhenyu, "Automatic eddy extraction from sst imagery using artificial neural network". the international 
archives of the photogrammetry, remote sensing and spatial information sciences. Volume XXXVII. Part B6b. Page(s): 279—282, 2008.

[11] B. Lemonnier, R. Delmas, C. Lopez and E. Duporte, "Multiscale analysis of shapes applied to thermal infrared sea images". Proc. Ocean'94 OSATES. Volume 3. Page(s): 319-322, 1994.

[12] A.I. Aleksanin and M.G. Aleksanina, "Quantitative Analysis of Thermal Sea Surface Structures on NOAA IR-Images". Proc. of the Int. Symposium CREAMS'2000. Page(s): 158-1651, 2000.

[13] H. Thonet, B. Lemonnier and R. Delmas, "Automatic segmentation of oceanic eddies on AVHRR thermal infrared sea surface images". Oceans. Volume 2. Page(s): 1122-1127, 1995.

[14] A. Turiel, V. Nieves, E. Garcia-Ladona, J. Font, M.H. Rio, and G. Larni$\mathrm{col}$, "The multifractal structure of satellite sea surface temperature maps can be used to obtain global maps of streamlines". Ocean Sci. Discuss., Volume 6. Page(s): 129-151, 2009.

[15] J.A.T. Arriaza, F.G. Rojas and M.P. Canton "Competitive neuralnet-based system for the automatic detection of oceanic mesoscalar structures on AVHRR scenes". IEEE Transactions on Geoscience and Remote Sensing, Volume 41, Issue 4. Page(s): 845—852, 2003. 